Article

\title{
Porous Silicon Based Resonant Mirrors for Biochemical Sensing
}

Edoardo De Tommasi ${ }^{1, *}$, Luca De Stefano ${ }^{1}$, Ilaria Rea ${ }^{1,2}$, Valentina Di Sarno ${ }^{1,2}$, Lucia Rotiroti ${ }^{1,3}$, Paolo Arcari ${ }^{4}$, Annalisa Lamberti ${ }^{4}$, Carmen Sanges ${ }^{4}$ and Ivo Rendina ${ }^{1}$

${ }^{1}$ Institute for Microelectronics and Microsystems - Unit of Naples - National Council of Research, Via P. Castellino 111, 80131 Napoli, Italy.

2 Department of Physical Sciences, University of Naples "Federico II", Via Cinthia, 80126 Naples, Italy

${ }^{3}$ Department of Organic Chemistry and Biochemistry, University of Naples "Federico II", Via Cinthia, 80128 Napoli, Italy

4 Department of Biochemistry and Medical Biotechnologies, University of Naples "Federico II", Via S. Pansini 5, 80131 Napoli, Italy.

* Author to whom correspondence should be addressed: edetommasi@na.imm.cnr.it

Received: 6 June 2008; in revised form: 6 October 2008 / Accepted: 21 October 2008 / Published: 23 October 2008

\begin{abstract}
We report on our preliminary results in the realization and characterization of a porous silicon (PSi) resonant mirror (RM) for optical biosensing. We have numerically and experimentally studied the coupling between the electromagnetic field, totally reflected at the base of a high refractive index prism, and the optical modes of a PSi waveguide. This configuration is very sensitive to changes in the refractive index and/or in thickness of the sensor surface. Due to the high specific area of the PSi waveguide, very low DNA concentrations can be detected confirming that the RM could be a very sensitive and labelfree optical biosensor.
\end{abstract}

Keywords: DNA Optical Biosensors, Porous Silicon, Resonant Mirrors.

\section{Introduction}

Optical label-free biosensors are ideal candidates for high throughput screening in the prevention of bio-threat agents or social illnesses: all the different configurations proposed in the literature, such as optical microcantilever [1], interferometric devices [2, 3], and also the recent photonic crystals 
geometries [4], show very high sensitivities in the recognition of specific molecules. The detection of analytes present in complex mixtures in ultra-low concentration, such as viruses in human blood, is possible due to two key factors: the utilization of bioprobes, properly linked on the sensor surface, and an optimized design to maximize the interaction between the biological matter and the electromagnetic field.

Resonant Mirrors (RMs) are optical sensors which exploit the evanescent fields features to probe changes in refractive index and thickness taking place on their surface after exposure to gaseous or liquid substances. From the optical point of view, RMs are refractometric sensing devices similar to grating coupler sensors: they are characterized by the high sensitivity typical of waveguiding devices, and, on the other hand, by the simple scheme of Surface Plasmon Resonance (SPR) sensors [5]. In $\mathrm{RMs}$, in fact, incident light gives rise to an evanescent wave at the interface between a prism and an optical waveguide; the coupling of light into the waveguide occurs only at those specific incident angles for which the propagation constant of the evanescent wave matches that of a waveguide mode. This matching produces a dip in the angular spectrum of the reflected light, so that each change in the refractive index and-or in thickness at the sensor surface produces a corresponding shift in the position of the dip $[5,6]$.

In this work, we report on the realization of a porous silicon (PSi) based RM prototype on chip, whose modal properties and characteristic angular resonances have been both numerically computed and experimentally measured.

PSi is by far one of the most intriguing material in optical sensing: the refractive index is widely tuneable, namely between the silicon refractive index and that of the air, and its specific surface is very large, up to $500 \mathrm{~m}^{2} \mathrm{~cm}^{-3}$. Due to these characteristics, lot of optical structures, such as rugate filters [7] and microcavities [8] have been proposed in literature for biosensing. Recently, a PSi waveguide biosensor have been theoretically and experimentally demonstrated [9]. We have studied and reported in this paper the feasibility of such biosensor for DNA-DNA hybridization experiment.

Figure 1. Experimental setup. DL: diode laser emitting s-polarized radiation at $785 \mathrm{~nm}$; L: lens with focal length $\mathrm{f}=7.5 \mathrm{~cm}$; RM: resonant mirror; RS: rotation stage; PM: power meter on an independent rotation stage.

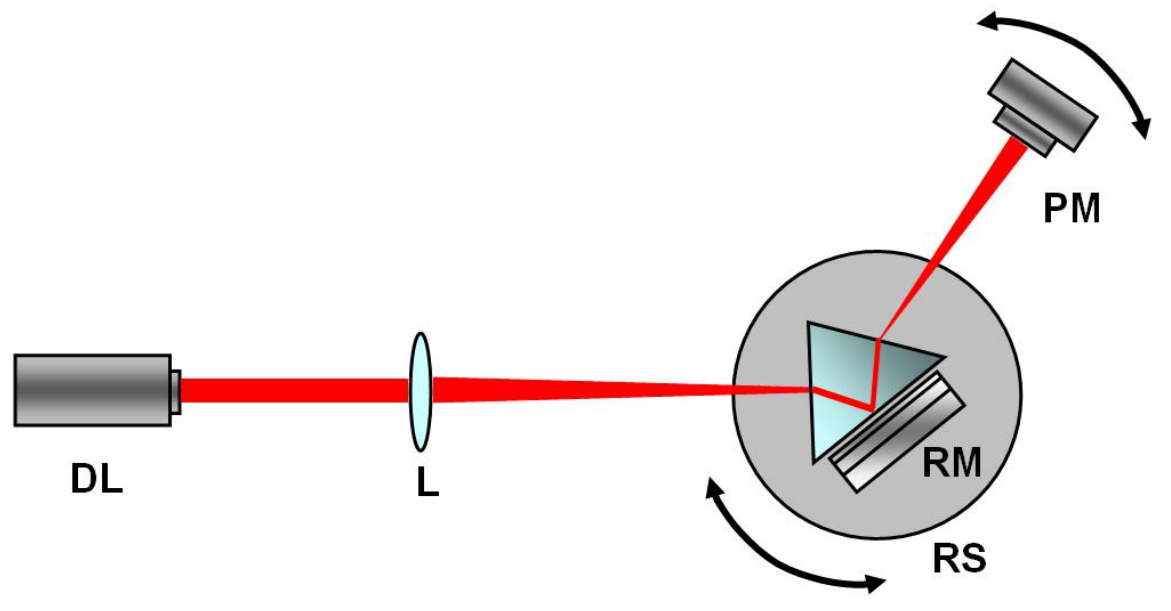




\section{Experimental}

A highly doped $\mathrm{p}+$-silicon, $<100>$ oriented, $0.01 \Omega \cdot \mathrm{cm}$ resistivity, $400 \mu \mathrm{m}$ thick was used as substrate in the waveguide fabrication. The structure was obtained by electrochemical etching of crystalline silicon in a HF-based solution ( $50 \mathrm{wt}$. \% HF:ethanol = 3:7) at room temperature. A current density of $10 \mathrm{~mA} / \mathrm{cm}^{2}$ was applied for $18.9 \mathrm{~s}$ to produce the core layer of thickness $2.5 \mu \mathrm{m}$ and porosity $65 \%$, while a current density of $109 \mathrm{~mA} / \mathrm{cm}^{2}$ was applied for $13.6 \mathrm{~s}$ in the case of the cladding layer with thickness $2.5 \mu \mathrm{m}$ and porosity $78 \%$. These porosities correspond to a core and cladding refractive indexes of 1.749 and 1.384, respectively, calculated by the Bruggeman model [10] at a wavelength of $785 \mathrm{~nm}$. The device was then fully oxidized in pure $\mathrm{O}_{2}$ by a two step thermal treatment $\left(400^{\circ} \mathrm{C}\right.$ for 30 $\min$ and $900{ }^{\circ} \mathrm{C}$ for $15 \mathrm{~min}$ ).

In Figure 1 a wide view of our experimental set-up is shown. RM is mounted on a rotation stage (Melles Griot, model 07 TRS 501); the light source is a diode laser emitting s-polarized radiation at $\lambda=785 \mathrm{~nm}$ (CrystaLaser, model RCL-080-785-5); the laser beam exiting the prism is collected by a photometer (Newport, model 1830-C). The waveguide has been brought in close contact with one side of an SF6 prism $(n=1.784)$, still allowing the presence of a sub-micrometric air layer acting as a coupling zone between the prism and the waveguide.

The covalent bond of the DNA single strand (5'-GGACTTGCCCGAATCTACGTGTCC-3') on the PSi surface is based on a three steps functionalization process constituted by a chemical passivation of the surfaces, as it is shown in Figure 2 (all the chemicals used in the present study were from Sigma). We have treated the surfaces with a proper chemical linker, the aminopropyltriethoxysilane (APTES). Samples have been rinsed by dipping in a $5 \%$ solution of APTES and a hydroalcoholic mixture of water and methanol (1:1) for $20 \mathrm{~min}$ at room temperature. After the reaction time, we have washed the chips by deionized water and methanol, and dried in $\mathrm{N}_{2}$ stream. The silanized devices were then baked at $100{ }^{\circ} \mathrm{C}$ for $10 \mathrm{~min}$. To create a surface able to link the amino group of the biological probes, we have thus immersed the chips in a $2.5 \%$ glutaraldehyde (GA) solution in $20 \mathrm{mM}$ HEPES buffer ( $\mathrm{pH} \mathrm{7.4)} \mathrm{for} 30 \mathrm{~min}$, and then rinsed it in deionized water and finally dried in $\mathrm{N}_{2}$ stream. The GA reacts with the amino groups on the silanized surface and coats the internal surface of the pores with another thin layer of molecules. All these reaction steps have been monitored by means of FT-IR spectroscopy to verify the presence of the characteristic peaks of the organic linkers. The main characteristic peaks of Si-O-Si $\left(1040 \mathrm{~cm}^{-1}\right)$ and Si-OH bonds $(3740$ and $1646 \mathrm{~cm}^{-1}$ ) are present, after oxidation, on the PSi. After the silanization process, the APTES characteristic peaks of the ethylic (at 1626, 1529 and $1379 \mathrm{~cm}^{-1}$ ), amino (at $1060 \mathrm{~cm}^{-1}$ ) and also the $\mathrm{CH}$ (at 2908 and $2851 \mathrm{~cm}^{-1}$ ) groups are well evident. Finally, after the GA treatment, the characteristic imine group (at $1627 \mathrm{~cm}-1$ ) due to the reaction with APTES is easily recognized.

The PSi surface was covered overnight at room temperature with a $50 \mu \mathrm{M}$ DNA probe solution (30 $\mu \mathrm{L})$. For each measurement, complementary (5'-GGACACGTAGATTCGGGCAAGTCC-3') and noncomplementary (5'-CACTGTACGTGCGAATTAGGTGAA-3') DNA (10 $\mu \mathrm{L})$ were spotted on the chip and incubated for two hours. Before optical measurements, all samples have been extensively rinsed in deionized water to remove the excess of biological matter. 
Figure 2. Schematic of the functionalization process, from the oxidized PSi chip to the covalent attachment of the DNA single strands.

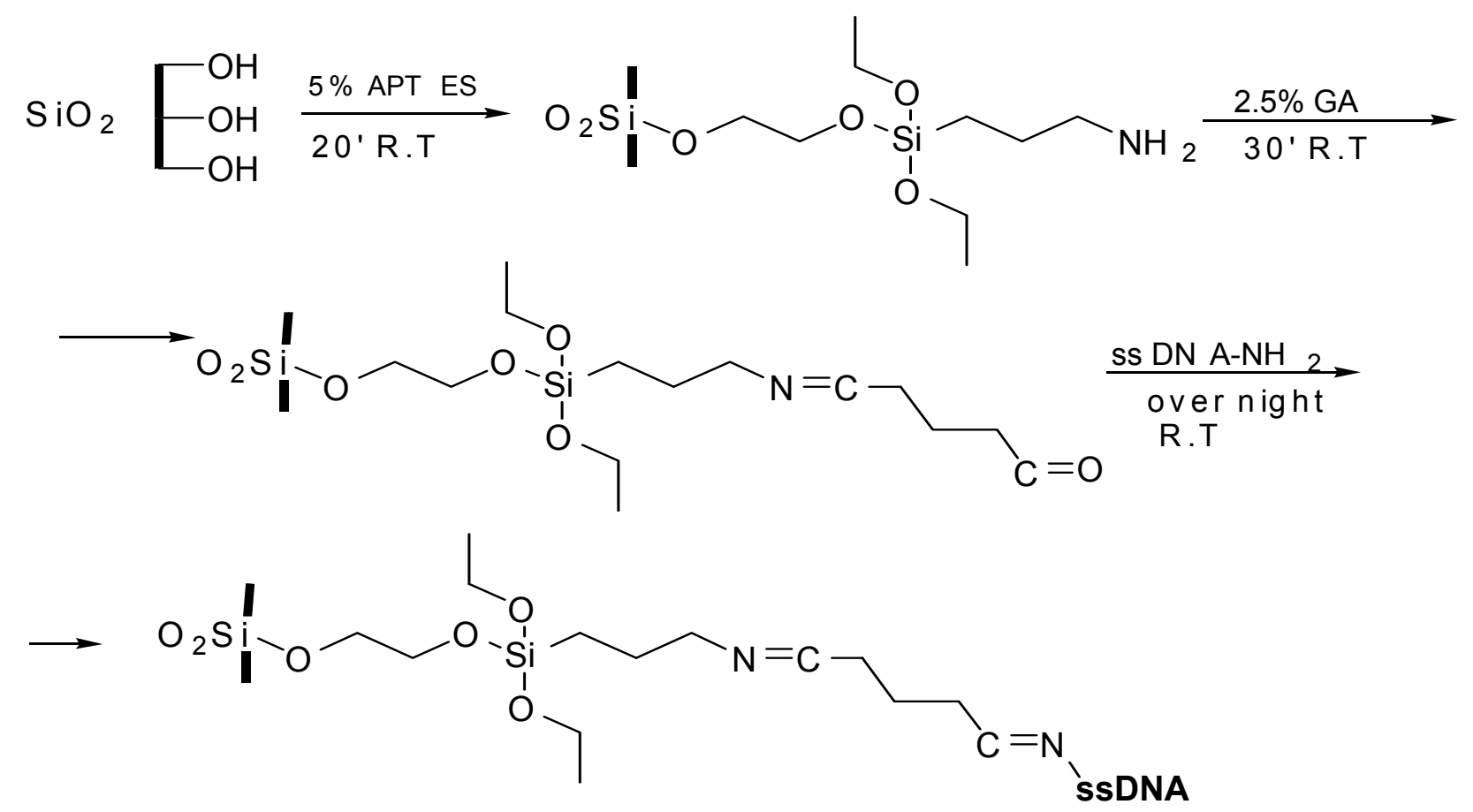

\section{Results and Discussion}

The PSi waveguide, once oxidized in order to reduce the scattering losses [11] and to allow the bond with biological linkers, has been characterized by means of " $m$ lines" technique, which determines the modal behavior of the waveguide at different wavelengths and estimates the refractive indices $n_{c}$ and $n_{b}$ for the core an the buffer layers [12]. In Figure 3, is reported a standard m-lines spectrum due to the excitation of four adjacent modes in transverse electric (TE) polarization of the electromagnetic field at $785 \mathrm{~nm}$ : by measuring the coupling angles position is possible to quantify the core and cladding thicknesses and refractive indexes. We have obtained the refractive indexes of core and buffer and the thickness of the core equal to $n_{c}=1.370 \pm 0.001, n_{b}=1.18 \pm 0.01$, and $\mathrm{d}_{\mathrm{c}}=2.71 \pm$ $0.01 \mu \mathrm{m}$ respectively. It is important to notice the strong reduction of the refractive indexes after the thermal oxidation process, mainly due to the substitution of silicon with silicon dioxide.

The close contact between the prism and the waveguide still allows the presence of a sub-micrometric air layer where the evanescent fields coming from the prism and the waveguide can overlap, thus transferring the light energy from the prism to the waveguide. The thickness of this air layer strongly affects the efficiency of the coupling of the incoming light into the waveguide. In Figure 4, some simulations of RM spectra corresponding to different air thicknesses are shown: the lower the thickness, the stronger the coupling of the evanescent wave with the waveguide. The dips present in the total reflection zone (i.e. for incident angle $\theta_{i}>34^{\circ}$ ), correspond to the characteristic modes propagating in the waveguide. The number of these modes can be obtained from the well known relation $M=\frac{2 d}{\lambda} \sqrt{n_{c}^{2}-n_{b}^{2}} \approx 5$, where $d$ stands for the thickness of the waveguiding layer. 
Figure 3. Resonant peaks of the waveguide, corresponding to four guided modes in TE polarization at $785 \mathrm{~nm}$, after functionalization with APTES and GA.

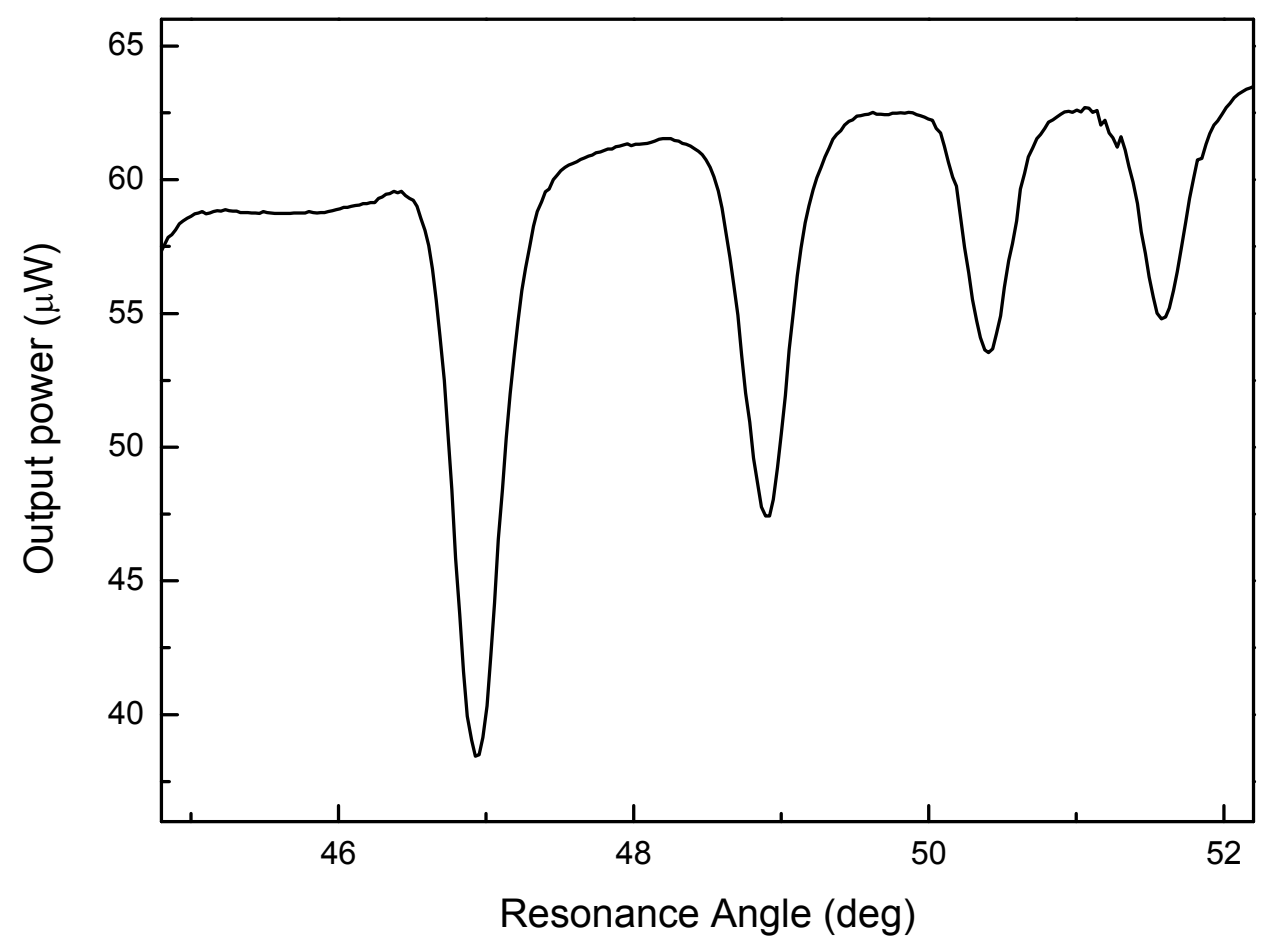

Figure 4. Numerical simulations of RM spectra as a function of the air gap thickness

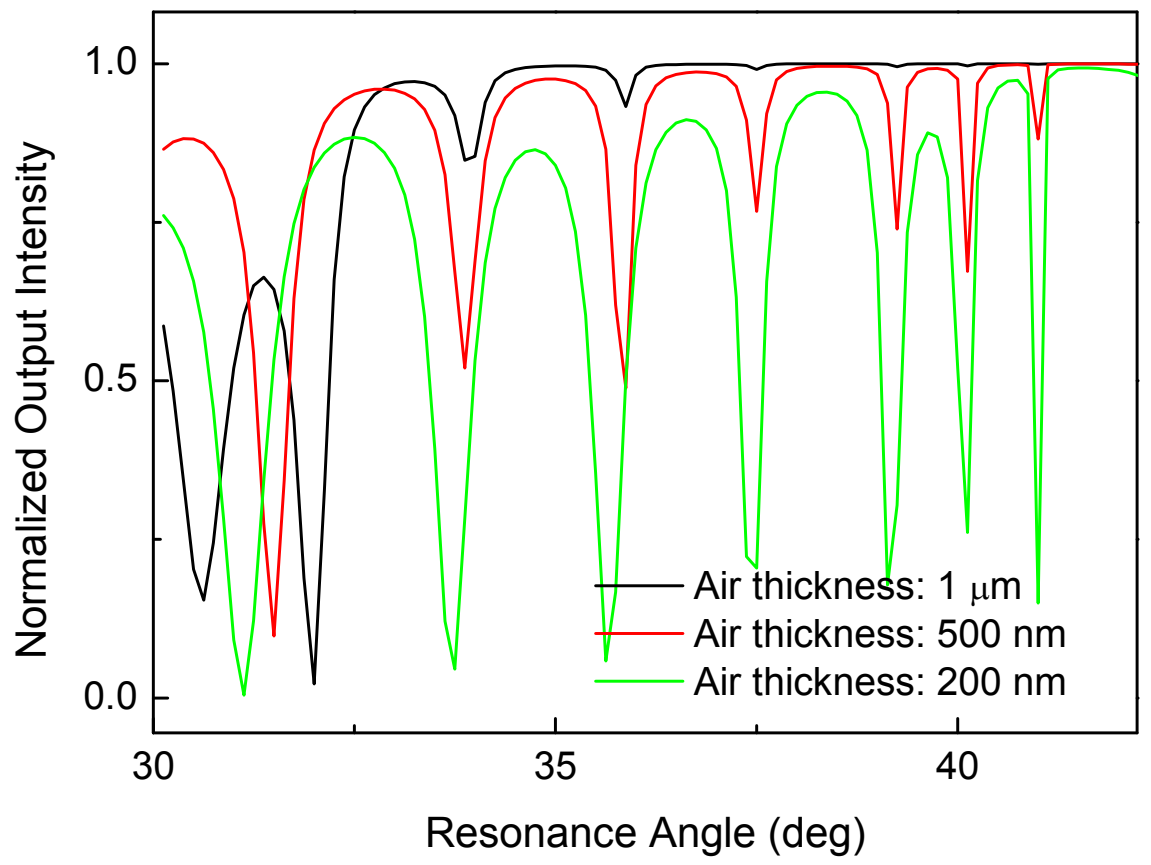

In Figure 5, the results of angular measurements after incubation with probe DNA and after two steps of the DNA hybridization process at two different concentrations are reported: the resonance shifts confirm the effectiveness of the DNA binding. 
Figure 5. PSi RM resonances after functionalization with probe DNA and after two DNA hybridization steps: the coupling angle shifts demonstrate the molecular interaction between the probe DNA and its complementary target at different concentration.

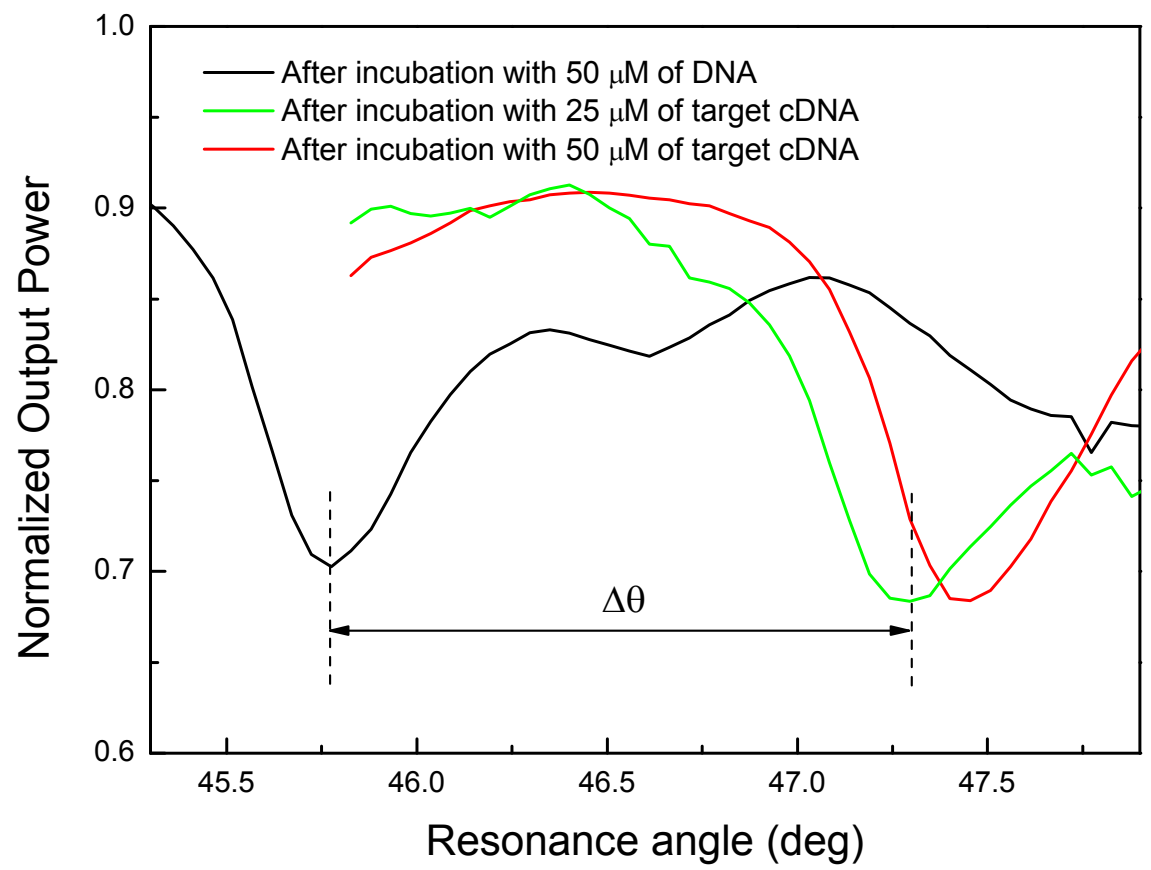

The coupling angle shifts as a function of the concentration of the complementary DNA, as it can be seen in Figure 6. The high efficiency of hybridization is confirmed by the fact that, after exposure to $50 \mu \mathrm{M}$ of complementary DNA, the sensor response curve reaches a plateau, corresponding to a saturation of almost all of the active sites. The experimental points can be well fitted $\left(\mathrm{R}^{2}=0.99, \chi^{2}<1\right)$ by a molecular growth exponential curve: from this fit a sensitivity of $0.13 \pm 0.02 \mathrm{deg} / \mu \mathrm{M}$ can be estimated. Since the angular resolution of the experimental setup is $0.01 \mathrm{deg}$, we can estimate, for our measuring system, a technical limit of $77 \pm 12 \mathrm{nM}$. This value, that has been estimated from experimental data, is of the same order of magnitude of that reported in ref. [13], where a detection limit of $50 \mathrm{nM}$ is predicted on the basis of numerical simulations and under the assumption of an optimal $50 \%$ probe coverage of the PSi surface. On exposure to the non complementary DNA sequence the angular resonance does not undergo any detectable shift, i.e. no shifts more than $0.01 \mathrm{deg}$ can be observed. The width of the resonances (e.g. FWHM of about 0.37 deg for the first resonance) can be considerably reduced, thus increasing the sensitivity of the sensor, by reducing the waveguide losses, mainly due to the roughness of the structure. A dramatic reduction of these losses can be achieved, for example, by performing the etching process at low temperatures [14] and/or by making use of a mono-modal waveguide.

\section{Conclusions}

We have experimentally confirmed the ability of a PSi RM to detect a DNA-DNA hybridization process with very high sensitivity. The use of PSi technology in the fabrication of the waveguide, which is the heart of a RM sensor, allows the presence of a high specific-surface available for 
interaction with great amounts of analytes. We have demonstrated that our measuring system is characterized by a technical limit of about $80 \mathrm{nM}$ of DNA, in good agreement with previously published theoretical calculations [13]. Next efforts will be aimed at the fabrication of a free-standing waveguide (no crystalline silicon substrate), which, in conjunction with a flow delivery system, could allow to reduce considerably the time of incubation of the DNA probe and targets over the functionalized surface.

Figure 6. Resonance shifts of the waveguide mode coupling angle as a function of the DNA concentration.

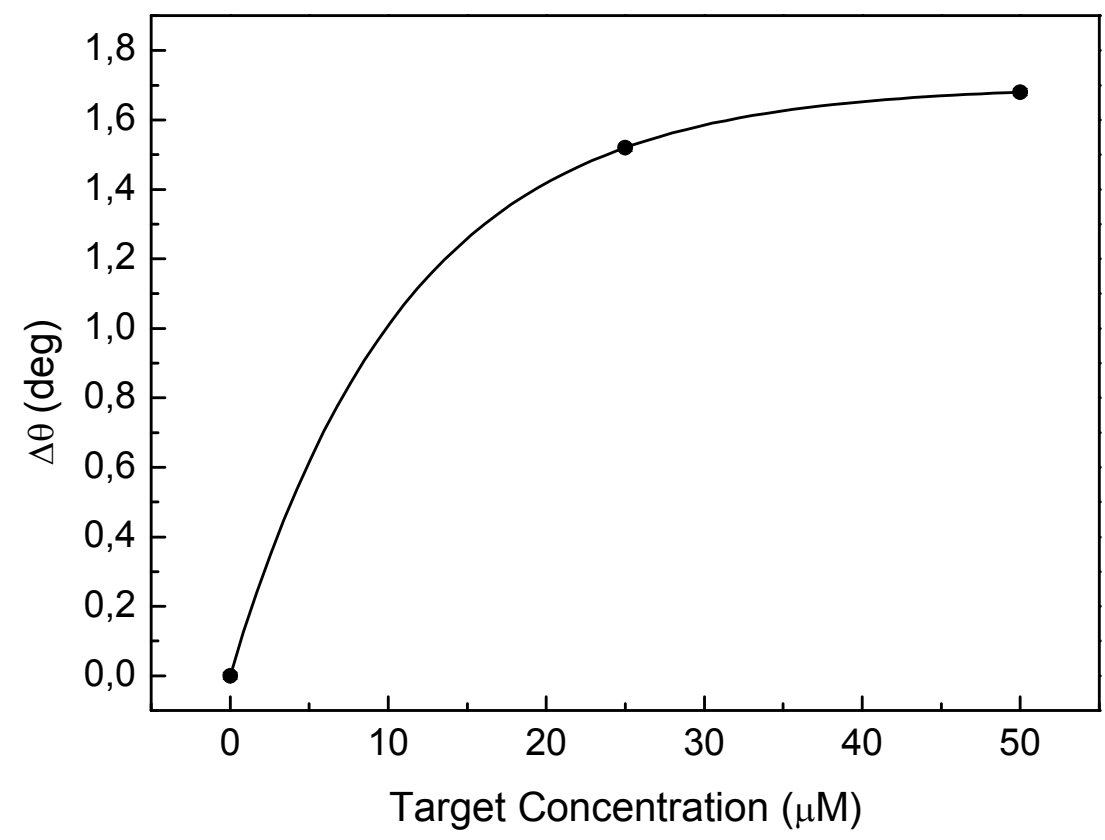

\section{Acknowledgements}

This work is partially supported by the FIRB Italian project RBLA033WJX_005.

\section{References and Notes}

1. Lechuga, L.M.; Tamayo, J.; Álvarez, M.; Carrascosa, L.G.; Yufera, A.; Doldán, R.; Peralías, E.; Rueda, A.; Plaza, A.J.; Zinoviev, K.; Domínguez, C.; Zaballos, A.; Moreno, M.; Martínez-A, C.; Wenn, D.; Harris, N.; Bringer, C.; Bardinal, V.; Camps, T.; Vergenègre, C.; Fontaine, C.; Díaz, V.; Bernad, A. A highly sensitive microsystem based on nanomechanical biosensors for genomics applications. Sensor. Actuat. B-Chem. 2006, 118, 2-10.

2. Pacholski C.; Sartor M.; Sailor M. J.; Cunin F.; Miskelly G. M. Biosensing using porous silicon double-layer interferometers: Reflective interferometric fourier transform spectroscopy. J. Am. Chem. Soc. 2005, 127, 11636-11645.

3. De Stefano, L.; Rotiroti, L.; Rea, I.; Rendina, I.; Moretti, L.; Di Francia, G.; Massera, E.; Lamberti, A.; Arcari, P.; Sangez, C. Porous silicon-based optical biochips. J. Opt. A-Pure Appl. Op. 2006, 8, S540-S544. 
4. Lee, M.R.; Fauchet, P.M. Two-dimensional silicon photonic crystal based biosensing platform for protein detection. Opt. Express 2007, 15, 4530-4535.

5. Cush, R.; Cronin, J.M.; Stewart, W.J.; Maule, C.H.; Molloy, J.; Goddard, N.J. The resonant mirror: a novel optical biosensor for direct sensing of biomolecular interactions. I: Principle of operation and associated instrumentation. Biosens Bioelectron. 1993, 8, 347-353.

6. Baldini, F.; Chester, A.N.; Homola, J.; Martellucci, S. Optical Chemical Sensors. Springer: Dordrecht, The Netherlands, 2006.

7. Chapron, J.; Alekseev, S.A.; Lysenko, V.; Zaitsev, V.N.; Barbier, D. Analysis of interaction between chemical agents and porous Si nanostructures using optical sensing properties of infra-red Rugate filters. Sensor. Actuat. B-Chem. 2007, 120, 706-711.

8. Ouyang, H.; Chrtistophersen, M.; Viard, R.; Miller, B.L.; Fauchet, P.M. Macroporous silicon microcavities for macromolecule detection. Adv. Funct. Mater. 2005, 15, 1851-1859.

9. Saarinen, J.J.; Weiss, S.M.; Fauchet, P.M.; Sipe, J.E. Optical sensor based on resonant porous silicon structures. Opt. Express 2005, 13, 3754-3764.

10. Khardani, M.; Bouaïcha, M.; Bessaïs, B. Bruggeman effective medium approach for modelling optical properties of porous silicon: comparison with experiment. Phys. Stat. Sol. 2007, 4, 19861990.

11. Pirasteh, P.; Charrier, J.; Dumeige, Y.; Haesaert, S.; Joubert P. Optical loss of porous silicon and oxidized porous silicon planar waveguides. J. Appl. Phys. 2007, 101, 083110.

12. Ulrich, R.; Torge, R. Measurement of thin film parameters with a prism coupler. Appl. Optics 1973, 12, 2901-2908.

13. Rong, G.; Najmaie, A.; Sipe, J. E.; Weiss, S. M. Nanoscale porous silicon waveguide for label-free DNA sensing. Biosens. Bioelectron. 2008, 23, 1572-1576.

14. Descrovi, E.; Frascella, F.; Sciacca, B.; Geobaldo, F.; Dominici, L.; Michelotti, F. Coupling of surface waves in highly defined one-dimensional porous silicon photonic crystals for gas sensing applications. Appl. Phys. Lett. 2007, 91, 241109.

(C) 2008 by the authors; licensee Molecular Diversity Preservation International, Basel, Switzerland. This article is an open-access article distributed under the terms and conditions of the Creative Commons Attribution license (http://creativecommons.org/licenses/by/3.0/). 